\title{
Disodium Cromoglycate in the Management of Asthma
}

\begin{abstract}
"Medicine Today" is the television series for doctors produced by the B.B.C. Advice on the preparation of the programme is given by the Association for the Study of Medical Education.

The programme on B.B.C.-2 on 16 fuly was on the subject of common patterns of respiratory infections in children. Printed below is an article prepared with the help of expert contributors to complement this television programme, which will be repeated on B.B.C.-1 on 23 fuly at about 11 p.m.
\end{abstract}

Disodium cromoglycate is the sodium salt of 1,3-bis-(2 carboxychromon-5-yloxy)-2-hydroxypropane. Cromoglycate is not a bronchodilator nor an anti-inflammatory agent. It has no sympathomimetic, anti-histamine, or corticosteroid-like effects. In vitro, the drug inhibits the release of various mediators of anaphylaxis arising from the interaction of antigen with reagin type antibodies-that is, "Type I," immediate allergic reactions. Cromoglycate causes a reduction in the contractile response of human bronchial smooth muscle exposed to passively sensitized and shocked human lung, a system which has been used to simulate an attack of allergic asthma. Cromoglycate can also inhibit the release of histamine and slow reacting substance of anaphylaxis (S.R.S.-A) from portions of human lung passively sensitized with human reaginic serum following exposure to specific antigens in vitro ${ }^{1}$.

There is also evidence from in vivo human studies of activity against a number of type III reactions. Experiments on volunteers have shown the drug to inhibit the effects of inhalation of antigens both on immediate asthmatic reactions and on late asthmatic and alveolar reactions, presumably precipitin mediated.

A very small proportion of the drug (less than $0.5 \%$ ) is absorbed after oral administration and possibly up to $5 \%$ after inhalation, representing the powder reaching the periphery of the lungs. When given by inhalation it has few general pharmacological effects. The absorption, distribution, and excretion have been studied mainly in laboratory animals. The absorbed compound is rapidly eliminated unchanged in the urine and bile and no accumulation has been detected.

\section{Toxicity}

Clinical trials are known to have involved only about 300 patients, for possibly 2,500 patient-months, and so far no toxic effects have been reported. A few patients have complained of some irritation of the throat on inhaling the capsule-but this has occurred both with the active preparation and with the lactose vehicle. In animal experiments no toxic effects were found in 90-day inhalation studies. The $\mathrm{LD}_{50}$ on parenteral administration to small laboratory animals was commonly between 2,000 and $4,000 \mathrm{mg} . / \mathrm{kg}$. In vitro tests showed that a concentration of at least $5 \mathrm{mg}$. $/ \mathrm{ml}$. was required to produce effects on the morphology of H.E. p2 cells and chick embryo fibroblast cells. In anaesthetized dogs, however, intravenous injection of doses of about $8 \mathrm{mg}$. $/ \mathrm{kg}$. caused immediate collapse and transient apnoea with rapid recovery; doses as small as $10 \mu \mathrm{g} . / \mathrm{kg}$. elicited reflexes from the coronary and pulmonary circulations. These effects did not occur in other animals tested. No teratogenic effects were seen in rabbits in which the compound was given intravenously daily throughout pregnancy in doses up to $250 \mathrm{mg} . / \mathrm{kg} .^{1}$ At least five healthy infants are known to have been born to mothers taking disodium cromoglycate throughout pregnancy. ${ }^{2}$

\section{Therapeutic Use}

Allergic Bronchial Asthma.-The earliest report ${ }^{3}$ of the use of cromoglycate was in asthmatic patients known to be sensitive to specific antigens. For several hours after inhalation of the drug the patients were protected against asthmatic responses to inhalation of the antigen. However, the drug is ineffective if it is inhaled even a few minutes after the antigen has been inhaled. The indications, therefore, are that the drug should be effective when used prophylactically to prevent or minimize episodes of bronchospasm in allergic subjects.

But it has not always been easy to identify extrinsic allergens. Recent work ${ }^{4}$ suggests that many patients may be reacting to the high population of mites which has been found in house dust. Some patients at present considered to have intrinsic asthma may be reclassified as a result of this work. Intrinsic asthma is considered to be due to hypersensitivity to some substance being produced in the body-probably in the lungs, and possibly often of bacterial or other infective origin. In these patients there may be a continuous or intermittent production of allergen, independent of any recent inhalation,'which may continue during the course of a severe asthmatic attack. So patients with allergic asthma of extrinsic or intrinsic type might be expected to benefit from a prophylactic drug, even if it is taken after the onset of an acute attack; and it would not necessarily be logical to withhold the drug because the patient already has bronchospasm.

Allergic Alveolitis.-An interesting new development has been the discovery by Pepys et al. ${ }^{6}$ that the drug also inhibits the type III reaction to certain inhaled substances including those implicated in bird fancier's lung and in aspergillosis. Since clinical exposure is usually easily defined, and the disease is often economically important to the patient, the drug may prove especially valuable to sufferers from these diseases. Clinical information already available suggests that cromoglycate has been particularly effective in asthmatic subjects with precipitating antibodies to aspergillus in their blood.

\section{Clinical Trials}

Four double blind, cross-over controlled trials have been carried out in Britain and two in the U.S.A.

In each trial a mixture of disodium cromoglycate and $0.1 \mathrm{mg}$. isoprenaline sulphate was compared with lactose $+0.1 \mathrm{mg}$. isoprenaline. Howell and Altounyan ${ }^{7}$ described a study of 10 patients with severe chronic allergic asthma. All derived some subjective benefit from the drug, and sequential analysis of the results showed a highly significant difference in favour of the drug. There was much less effect of the drug on the F.E.V.. ; only four out of ten subjects benefited by this criterion. This was the main criticism made by Grant et al.,8 who found benefit in only one out of seven patients, and even in this subject the F.E.V..$_{1}$ improved by only $12 \%$. These workers also carried out a single blind trial with poor results, finding good response to the drug in only 11 out of 32 cases. However, Woolcock and Read $^{9}$ as well as Howell and Altounyan ${ }^{10}$ do not agree that the measurement of the F.E.V.. alone is a reliable measure of response in obstructive airways disease.

Grant and co-workers also emphasized the temporary nature of the response to disodium cromoglycate. Smith and Devey ${ }^{11}$ have since reported a double blind cross-over trial on 44 children over two months and obtained results similar to those of Howell and Altounyan. They noted no loss of clinical benefit over a further three-month period of open treatment with the drug. In addition, of 20 patients who had not shown improvement after one month of treatment, six benefited after a further three months. These authors felt that the reduction and successful replacement of steroid therapy in many cases might prove to be among the greatest benefits to be expected. Kennedy ${ }^{12}$ also suggested that improvement in 45 out of 62 
patients given disodium cromoglycate double blind showed the drug to have a considerable advantage over the placebo. A further trial ${ }^{13}$ of 21 patients with asthmatic symptoms was designed to last for three months and to provide three periods of two weeks' exposure to the drug and two weeks to the placebo. This trial also showed that disodium cromoglycate was significantly better than placebo in suppressing asthma and cough and that in those patients who used a bronchodilator inhaler usage was significantly lower on cromoglycate than on placebo. Steroid dosage was on average halved, though the difference was not statistically significant. Analysis of the patients in this trial with a view to determining the type of patient likely to benefit from the drug confirmed that those who had most evidence of allergy were more likely to benefit than those with considerable evidence of bronchitis. Further confirmation of these generally favourable results in allergic asthma have come from two sources"15 in the U.S.A. A longer "open" trial by Altounyan and Howell ${ }^{16}$ on 100 patients again confirmed that non-allergic subjects did not benefit but the majority of allergic patients improved and the improvement was maintained for up to 28 months (average $14 \frac{1}{2}$ months).

While some patients, particularly young, clearly allergic subjects, respond dramatically to the drug, it seems that the majority of subjects show useful but more modest improvement.

The suggestion has been made ${ }^{17}$ that the sodium sulphate included in the placebo capsules in several trials might have been harmful to some patients, so exaggerating the benefit of cromoglycate, but Pepys et al..$^{5}$ have now shown that the effect of such a placebo inhaled in conjunction with a known potent antigen produces asthmatic reactions no different from those produced by antigen alone. In addition these authors have fully confirmed the original findings of Altounyan ${ }^{3}$ on the inhibition of asthmatic reactions to inhaled allergen.

\section{Present Indications for Use}

The drug is presented as a dry powder of which $20 \mathrm{mg}$. is contained in a gelatin capsule. A special inhaler ("Spinhaler," Fisons Ltd.) is available which punctures the capsule. The patient's own inspiratory effort then causes the capsule to be spun round and vibrated in the inspired air-stream. The "micronized" powder is thus inhaled into the lungs. A small quantity reaches the alveoli. The suggested dose is two to eight capsules per day, most patients having received four capsules per day. Because the dry powder may cause bronchospasm in some patients the capsule also contains $0.1 \mathrm{mg}$. isoprenaline sulphate-about the amount contained in one dose of a conventional pressurized aerosol of isoprenaline. There is practically no danger of accidental overdosage with isoprenaline, as it requires five or six deep breaths to empty a single capsule. As disodium cromoglycate appears at present to be of very low toxicity, it seems reasonable to increase the frequency of administration of the drug at times when symptoms are increasing on the grounds that increased antigen challenge may be present at these times.

Disodium cromoglycate must now be considered to have a place in the prevention or prophylactic amelioration of acute attacks in patients with allergic bronchial asthma. The effect may be limited in many patients, but in view of the now wellknown hazards of bronchodilators and of long-term steroid therapy, particularly in children, any reduction in dependence upon these drugs is worth while. A proportion of patients may be able to discontinue steroids, but many will require to continue them, though at a lower dose. In acute asthma cromoglycate does not supplant conventional treatment, including if necessary the use of large doses of steroids. ${ }^{18}$

The role of cromoglycate in the clinical management of patients with allergic alveolitis is as yet unknown. The work of Pepys and his colleagues shows, however, that disodium cromoglycate promises to be a valuable tool for the study of immunological mechanisms in respiratory allergic diseases.

\section{REFERENCES}

' Cox, J. S. G., Nature (Lond.), 1967, 216, 1328.

Altounyan, R. E C., Personal communication, 1968. 487.

Maunsell, K., Wraith, D. G., and Cunnington, A. M., Lancet, 1968, 1,1267 .

- Pepys, J., Chan, Moira, and Hargreave, F. E., Lancet, 1968a, 1, 1270.

- Pepys, J., Hargreave, F. E., Chan, Moira, and McCarthy, D. S., Lancet, in press, $1968 \mathrm{~b}$.

' Howell, J. B. L., and Altounyan, R. E. C., Lancet, 1967a, 2, 539.

- Grant, I. W. B., Channell, Sonia, and Drever, J. C. Lancet, 1967, 2, 673.

- Woolcock, A. J., and Read, J., Lancet, 1965, 2, 1323.

10 Howell, J. B. L., and Altounyan, R. E. C., Lancet, 1967b, 2, 777.

11 Smith, J. M., and Devey, G. F, Brit. med. Ұ., 1968, 2, 340.

12 Kennedy, M. C. S., Lancet, 1967, 2, 838.

12 Moran F. Bankier, J D. H., and Boyd, G., Lancet, 1968 in press

14 Lopez, M., Franklin, W., and Lowell, F. C., 24th Annual Mecring of the American Academy of Allergy, 1968.

is Chen, J. L., Moore, M. K., Norman, P. S., and Van Metre, T. B., jun., 24th Annual Meeting of the American Academy of Allergy, 1968.

16 Altounyan, R. E. C., and Howell, J. B. C., Med. Thorac, 1967, in press.

"Drug. Ther. Bull., 1968, 6, 10.

10 Brit. med. F., 1968, 2, 750.

\section{ANY QUESTIONS?}

\section{We publish below a selection of questions and answers of general interest.}

\section{Home-cooked}

Q.-Assuming the initial ingredients are identical, how does a bone-and-vegetable broth differ in terms of calories, trace eleinents, and vitamins when prepared at home, taken from a tin, or when dehydrated?

A.-A reliable answer to this question would require direct research into all the factors involved. It may be suspected, however, that there would be little difference in the nutritive values of the three forms of broth provided there were no gross losses or removals of material during cooking or storage.

Bone and vegetable broths are low in calories, which could be mainly contributed by barley or by globules of fat derived from the bone. In the home kitchen the calorie content of the broth may be reduced by skimming away the fat, particularly if this process is applied to cold soup hefore reheating it for a second meal, It seems probable that fat may be kept low in dehydrated soups so as to facilitate storage.

The duration of heating in cooking the soup may affect its content in vitamins of the $\mathbf{B}$ complex. Vitamin $\mathbf{B}_{1}$ is the most thermolabile member of this group. Vitamin $C$ is also unstable to heating, but vitamin $A$ in the form of carotene is little affected. In dehydrated soup partial losses of vitamin may occur during storage, but these losses may be retarded by wrappings impermeable to air or by packing in an inert gas. Deterioration in flavour is probably a good guide to the extent of vitamin losses.

In tinned soups the vitamins are probably more stable than in dehydrated soups of only average quality. Trace elements will not be lost in any form of soup, provided that the liquor in which the vegetables are cooked remains part of the soup stock and is not discarded. This cause of loss would be unlikely in home-made soup, but it might possibly occur in the manufacture of dehydrated soups. The absorption of some nutrients, and particularly carotene, may be influenced by the state of subdivision of the vegetables, particularly carrots.

Though the terms of the question require that soups made from the same starting materials should be compared, this equality in the amounts of starting materials probabiy seldom holds good in practice. Cheap and tasty home-made broth may be eaten in large platefuls. Tinned or dehydrated broth will probably be less attractive in flavour, much more expensive, and supplied to the consumer in relatively small packings. Home-made soup may therefore be more nutritious than 